\title{
Problem of the degradation criteria for transportation construction elements
}

\author{
Albert Lantukh-Lyashchenko ${ }^{1}$, Artur Onishchenko ${ }^{1}$ and Oleksandr Davydenko, ${ }^{1, *}$ \\ ${ }^{1}$ National Transport University, 1, Mykhaila Omelianovycha - Pavlenka str., 01010, Kyiv, Ukraine
}

\begin{abstract}
The article is devoted to the pressing problem of managing the reliability and durability of transport constructions. Modern scientific approaches to assessing the technical condition of transport facilities based on an analysis of element degradation during the life cycle of operation considered. The article presents the advantages and disadvantages of the most common prediction models of the technical condition of building structures. Each model uses a characteristic criterion for degradation of building structures: load and unload cycles, rejection number of chlorides, intensity coefficient, a geometric parameter (parameters) of the crack in the material, reliability, etc. The problem of choosing the control parameter of the lifecycle model of the bridge elements is analyzed. The theoretical basis for the Markov phenomenological model of damage accumulation and the model of degradation based on the physical and mechanical characteristics of the material formulated. Depending on the stage of the life cycle, it is proposed to select the type of prediction model.
\end{abstract}

\section{Introduction}

Crucially important moment in the formulation of the model for estimating and predicting the life cycle is the adoption of the degradation criterion, that is, the degree of accumulation of damage. Theoretically, the criterion is an integral part of the fundamental problem of managing the reliability and durability of construction objects. In the theory of structures, at least ten different criteria in their physical essence are known. Let's call some of them.

In the structural mechanics from the 1950 's, the universal cumulative damage models were widely used, known as the "The Palmgren-Miner linear damage theory", which uses the principle of linear superposition of damage [1]. The criterion here is "load and unload cycles". This simple and transparent model has become widespread in mechanical engineering. To a lesser extent, the model was used to assess the life cycle of building elements in research works V. Bolotin, V. Chirkov. Obviously, to use the model it is necessary to have a reliable estimate of the number of load cycles, that is, to predict the service durability it is necessary to have full load history data [2, 3, 4]. As for the elements of highway bridges and other elements of structures, the required data is determined so approximately that the reliability of the model is lost. There are other disadvantages that

* Corresponding author: oleksandr.davydenko@ntu.edu.ua 
impede the application of the Palmgren-Miner model, such as, for example, ignoring the interaction effects of load cycles with small and large amplitudes.

Quite common today is models of the life cycle of reinforced concrete elements, in which the degradation of reinforced concrete is described by the cumulative criterions certain "rejection number of chlorides", penetrating through the protective layer on the capillary system and microcracks. The diffusion process of chloride here is described by the general laws of the analytic theory of diffusion, known as the equation of the Fick's first and second laws of diffusion $[5,6]$.

Another direction development of the models cumulative damage elements of building structures is based on fundamental researches of destruction mechanics. In Ukraine this is a study of the academician of the National Academy of Sciences (NASU) Panasyuk. In these works, the degradation material criterions of construction elements are "intensity coefficient" that are treated as physical constants of the material. Of course, models based on the classical damage mechanics theory are theoretically the most perfect, however, while they have not become widely used in the practical assessment of the service life elements of structures.

In the last 20-30 years it turns out that the universal, most effective degradation criterion elements of building structures is the "reliability parameter". Below we give a detailed justification for accepting reliability as a degradation criterion and an example - the corresponding model that is valid in the service system of Ukrainian highway bridges.

Finally, another widely used degradation criterion is the "geometric parameter (parameters) of the crack in the material" for estimating and predicting the life cycle. Geometrical parameters of cracks (width, length, depth) have been long used in models of degradation of metal elements of machines and mechanisms, structural steel member of buildings. In this work, we give an example of the life cycle model of reinforced concrete elements of transport constructions in which the degradation criterion is the width of the opening of cracks. $[7,8]$

\section{The reliability parameter is the universal degradation criterion}

\subsection{The idea of Markov's probabilistic chains}

The idea of using a scalar parameter - probability as a process criterion, the evolution of which in time is determined by probabilistic laws, belongs to an outstanding mathematician, a member of the Russian Academy of Sciences A.A. Markov. In a monograph 1st edition published in 1908 "The calculation of probabilities" for the first time fully published the Markov theory of processes (chains), it is the probability that it is the fundamental basis of the theory. Markov stochastic theory has spread rapidly in the world, primarily in banking and insurance affairs. In the 100 years since the publication, the stochastic theory of A.A. Markov intensively developed around the world and became the basis for models systems evolution in time, not only in banking, but also in all branches of knowledge - from economic management to medical predictions. However, it took more than 50 years for the theory has been begun used for models cumulative damage in the designs of machines, mechanisms, buildings and structures [9, 10]. In the past 30-40 years, phenomenological stochastic models describing the accumulation of damage as Markov process are recognized as a universal apparatus for describing the degradation failure of construction elements notwithstanding of material.

The expediency and the theoretical justification of the use of the reliability parameter as a criterion for degradation is obvious and proceeds, first of all, from the modern interpretation of the notion of reliability. Let us give a modern definition of the term in the 
formulation of European scientists. So, in the standard ISO 2394 [11], the definition is given in a very brief manner: "Ability of a structure or structural member to fulfil the specified requirements, during the working life, for which it has been designed". Similar definitions are also given in the USSR State Standard GOST 27751-88 and in the national regulatory documents Ukrainian State Building Standard DBN B.2.3-14:2006: Bridges and tubes and Ukrainian State Standard DSTU-N B V.2.3-23:2012: Instruction of estimate and prediction of technical state of highway bridges. These definitions differ fundamentally from the definition of the general technical standard USSR State Standard GOST 27.41089: Reliability in technology. Basic concepts.

A slightly broader notion of "reliability" is presented in Eurocode EN 1990:2002 [12]. Let's textual quotation: "Ability of a structure or a structural member to fulfil the specified requirements, including the design working life, for which it has been designed. Reliability is usually expressed in probabilistic terms". The note to the definition of the term indicates that reliability is at the same time a safety measure, service ability and durability of the design.

Reliability along with its main purpose - to characterize the level of safety of the building during the life cycle has a different function - serves as a tool for optimizing the quality of the project. So, in EN 1990:2002 [12], we find: "The actual frequency of failure is significantly dependent upon human error, which are not considered in partial factor design. Thus, reliability index does not necessarily provide an indication of the actual frequency of structural failure". The above definitions open the way to the use of reliability as a criterion for degradation - accumulation of damage, assessment of service ability in other terms.

In the most general form, the modern scientific substantiation of the problem is presented in the document of the Joint Committee on Structural Safety [13] - 2001 and in the monograph by Robert E. Melchers [10] - 1999, where reliability is treated as the probability of reaching a limit state for time t. For this, a function of a limit state, depending on time, is introduced:

$$
g(\boldsymbol{X}, t)=R(\boldsymbol{X}, t)-E(\boldsymbol{X}, t),
$$

where $R(\boldsymbol{X}, t)$ - summarize element resistance; $E(\boldsymbol{X}, t)$ - summarize load effect; $\mathbf{X}$ vector of basic variables; $t$ - time variable.

Then the reliability in terms of the function of limit state is expressed as:

$$
P(t)=\operatorname{Prob}[R(\boldsymbol{X}, t)-E(\boldsymbol{X}, t)<0] .
$$

Thus, dependence Eq. 2 technical condition, service ability is formulated as a concept associated with functional reliability. This general approach, in a slightly different form, was also described in monographs $\mathrm{V}$. Bolotin.

Below, as an example, the Markov model of accumulation of damage is presented in which reliability is the degradation criterion. The model was proposed by A. LantukhLyashchenko in 1999 and now has a reputation as a sufficient management apparatus for the safety maintenance, assessment and prediction of the technical condition of Ukrainian highway bridges.

\subsection{Markov phenomenological model of damage accumulation}

Let us consider degradation model of elements structures based on stochastic Markov processes. The failure system, which is the result of a building element wear, will be considered as a stream of random discrete events of the Markov chain and simulated by a 
process with "qualitative states". The role of the random variable plays the "random discrete state of the system" [9, 14, 15, 16, 17].

The element degradation model is established by the law of reliability in the function of time, thus set the apparatus prediction it's technical state. The model has two principal components: the phenomenological classification tables of discrete states and the function of degradation. The following four hypotheses are the theoretical basis of the model.

A. The criterion for the technical state of an element is the numerical parameter of reliability.

B. The life cycle of the element is divided into 5 technical states. Each of the states is described by a collection of quantitative and unformalized qualitative degradation indicators that characterize the element hierarchy of failures.

C. The process of degradation of an element during a life cycle is described by the discrete model of a random Markov process with continuous time.

D. Transition from one technical state to another is described as the Poisson process with discrete states and continuous time.

We formulate deterministic dependencies for a model in which wandering through discrete states is carried out in one direction only: from a state smaller, to a state with a larger number. We assume that the element is consistently in the discrete states $S_{1}, S_{2}$., $S_{n}$ and transitions from one technical state to another are carried out at moments of continuous time $t_{1}, t_{2}, \ldots, t_{n}$. The problem is to find probabilities of states $p_{1}(t), p_{2}(t), \ldots, p_{n}(t)$ where $n$ is the number of discrete states. The reliability of the system in each of the states is determined by the degradation process.

The Markov chain is a flow of sequential events with equally distributed time intervals. The graph of the flow is shown in Fig. 1 .

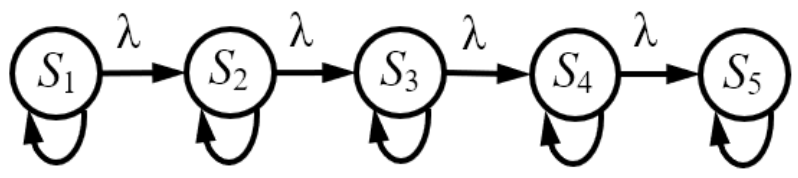

Fig. 1. The graph degradation process.

In the terms of the Markov process, the task is to find the unconditional probabilities of staying the system $S$ at some step $k$ in the state $S_{i}: k=1,2, \ldots, n$ :

$$
p_{i}(k)=\operatorname{Prob}\left[S(k)=S_{i}\right] ; k=1,2, \ldots, n ; i=0,1, \ldots n-1 .
$$

The probabilities $p_{i}(k)$ are expressed by the probabilistic probability of transition system $S$ in step $k$ to state $S_{j}$, provided that in step $k-1$ the system was in the state $S_{i}$ :

$$
p_{i j}(k)=\operatorname{Prob}\left[S(k)=S_{j} \mid S(k-1)=S_{i}\right] ; i, j=0,1, \ldots, n-1 .
$$

The evolution of the system is characterized by the density of the probability transition (hazard rate, degradation rate) $\lambda_{i, i+1}$. This parameter is the limiting relation of the system probability transition in time from the state $S_{i}$ to the state $S_{i, i+1}$ :

$$
\lambda_{i, i+1}=\lim \frac{p_{i, i+1}(\Delta t)}{\Delta t}
$$

where $p_{i, i+l}(\Delta t)$ - is the probability that the system will pass over time $\Delta t$ from the state $S_{i}$ to the state $S_{i, i+l}$.

The desired probabilities of the Markov chain $p_{1}(t), p_{2}(t), P_{n}(t)$ - the function of time is the probability that the system at the time $t$ is in the state $S_{i}$ and are determined from the 
system of ordinary differential equations with variables, in the general case, by coefficients. These are known Chapman-Kolmogorov equations describing the evolution of the discrete Markov process with continuous time:

$$
\frac{d p_{i j}(t)}{d t}=\sum \lambda_{i k} p_{k j}(t), \quad i, j, k=1,2, \ldots, n .
$$

These equations are joined by initial data

$$
p_{i j}(0)=\delta_{i j} \text {, }
$$

where $\delta$ - Kronecker symbol.

In addition, in the solution of the system differential equations the condition of valuation is used, which is a consequence of the fact that events of the Markov chain are incompatible and form a complete group:

$$
\sum_{i=1}^{n} p_{i}(t)=1
$$

The solution of the system of Eq. 6 under initial data Eq. 7 gives the matrix of probabilities transitions $p_{i k}(t)$, which describes the Markov chain. This matrix is often called shortened by a transition matrix.

The reliability of an element is calculated as the probability that at the time $t$ the element will exit from the state $k$, that is, the failure $k+1$ will occur:

$$
P_{i}(t)=\sum_{k=1}^{N} p_{k} p_{i k}(t),
$$

where $N$ - number of discrete states during the life cycle of the element; $p_{k}$ - reliability of the element assigned to it in a $k$-th discrete state; $p_{k}(t)$ - is the transition probability of the $k$ th discrete state obtained by the solution of the system of equations Eq. 6 under initial conditions Eq. 7.

As is clear from Eq. 9, in the general case of the transition probability, there is a square matrix of transitions of size $n$, where $n$ is the number of discrete states. Let us call it is $\mathbf{P}$. On the main diagonal of the matrix $\mathbf{P}$, the probability of the system delay in this state $S_{i}$ in step $k$ is on the side diagonals - the transition probability of the system from the state $S_{i}$ to the state $S_{j}-p_{i j}(\mathrm{k})$. Because that at each step the system can only be in one of two mutually exclusive states, the sum of all probabilities of one line is equal to one:

$$
\sum_{j=1}^{n} p_{i j}(k)=1
$$

Differential equations of the Markov chain Eq. 6, corresponding to the flow graph of Fig. 1, are written in the matrix form:

$$
\frac{d \mathbf{P}(i, t)}{d t}=\mathbf{P}(i, t) \cdot \mathbf{E}
$$

where $\mathbf{P}(i, t)$ - is the matrix of transition probabilities; $\mathbf{E}$ - is the matrix of hazard rates, are set as a constant $\mathbf{E}=\lambda$.

In the case of the model for the linear flow graph Fig. 1, where wandering in the states occurs in one direction - from a state with a smaller number to a state with a larger number, 
the matrix of the transition becomes triangular overdiagonal, little filled. An example of solving a system of equations along the linear flow graph is given below:

$$
\mathbf{P}=\left[\begin{array}{ccccc}
0.181 & 0.819 & 0 & 0 & 0 \\
0 & 0.330 & 0.670 & 0 & 0 \\
0 & 0 & 0.451 & 0.549 & 0 \\
0 & 0 & 0 & 0.551 & 0.449 \\
0 & 0 & 0 & 0 & 1
\end{array}\right] .
$$

Note that the described classical procedure for obtaining a transition matrix is not unique. Known techniques for obtaining a transition matrix are based on the historical data of the maintenance (for example [16, 18]), but this is another topic.

The search for the transition probabilities that contains the stochastic matrix $\mathbf{P}$ is dominant, the central place in the development of the degradation model of the construction element described by the discrete Markov process. When the matrix $\mathbf{P}$ is found, according to the known values of the conditional probabilities of the transition and the initial value of the absolute probability of staying the system in the state $S_{1}$, all other absolute probabilities are based on the recurrence formula:

$$
p_{j}(k)=\sum_{i=1}^{n} p_{i}(k-1) p_{i j}, k=1,2, \ldots, n ; j=1,2, \ldots, n .
$$

The degradation model establishes a connection between the reliability and the time in service of the element. The transition from one discrete state to another is described as a Poisson process with discrete states and continuous time. This is a special case of the Markov process. The integral distribution function $P(t)$ for the time $T_{n}$, which proceeds until all $n$ events of the process occur, has the form:

$$
P_{t}=1-P\left(T_{n}>t\right)=1-\sum_{k=0}^{n-1} \frac{(\lambda t)^{k} e^{-\lambda t}}{k !},
$$

where $\lambda$ - parameter of the process - the hazard rate; $k$ - discrete state number; $P(t)$ - is the probability that the element will go to state $k$ for time $t<T_{k}$.

For a case of a linear graph of five discrete states, the Eq. 14 has the form:

$$
P_{t}=1-0,008333 \cdot(\lambda t)^{5} e^{-\lambda t}
$$

Thus, with a given hazard rate $\lambda$, the Eq. 15 establishes the relationship between the reliability of the element $P_{t}$ in the $i$-th state and the time $t$, which has passed from the beginning of service to the state $i=2, \ldots, 5$.

The Markov stochastic model of damage accumulation as a result of natural wear is quite versatile and has a practical orientation as a tool for assessing the technical condition and prediction the remaining life of the structure. The model was found to be in demand in Ukraine and is now successfully used in the system of highway bridges service maintenance as a safety management tool, assessment and prediction of the technical state of transport constructions elements.

\section{The reliability parameter}


The alternative model of estimation and prediction of the life cycle of reinforced concrete elements presented here is based on the function of one parameter - the width of the opening of normal cracks. The width of the opening cracks, in turn, is a function of the physical parameters and mechanical characteristics of the element material. In such a statement, the model can be applied at all stages of the life cycle, from the design stage. The task is limited to reinforced concrete bending elements of the bridges.

The theoretical basis of the model contains two hypotheses:

A. The criterion for the technical state of an element is a numerical parameter of the width of the crack opening, which serves as a quantitative integral characteristic of the degradation process.

B. The width of the crack opening is sufficiently informative to construct the degradation law of the reinforced concrete element in the function of time.

The degradation process - the growth width of the crack opening will be described by one-dimensional differential equation:

$$
\frac{d A(t)}{d t}=\gamma A(t)
$$

where $A(t)$ - is the width of the opening cracks, the function of time; $\gamma$ - degradation rate, constant; $t-$ is the time.

The solution of the differential Eq. 16 has the form:

$$
A(t)=a_{0} e^{-\gamma t}
$$

where $a_{0}-$ is the initial value of the crack opening width, which is the constant of integration, which is determined from the initial data: at $t=0, A(t)=a_{0}$.

The initial value of the opening cracks width of the in the design is determined by the function of O.Ya. Berg:

$$
a_{0}=f_{\mathrm{a}}(H, R, E, \sigma, \Psi),
$$

where $H$-geometrical characteristics of the section; $R, E$ - physical and mechanical characteristics of the section; $\sigma$ - normal stress in a section; $\Psi-$ is the section factor. Parameters $(H, R, E, \sigma, \Psi)$ are determined in accordance with the requirements of the Ukrainian State Building Standard.

The design lifetime of $T$ will be obtained from the Eq. 17 by putting $A(t)=A_{\text {lim, }}$ where $A_{\text {lim }}$ is a scalar, the limit value width of the opening cracks in service:

$$
T=\frac{1}{\gamma} \ln \left(\frac{A_{\lim }}{a_{0}}\right)
$$

For use in the projection practice Eq. 17, we introduce a reliability factor of 5\% quantile of a normally distributed variable $T$ :

$$
T_{d}=T \cdot \gamma_{t}
$$

where $T_{d}$ - is the design lifetime of the $T$ element; $\gamma_{t}$ - coefficient of reliability of the life cycle time.

The reliability factor is determined by the function:

$$
\gamma_{t}=e^{0,8 \cdot \beta \cdot V_{R}-1,645 \cdot V_{Q}}
$$


where $\beta$ - the limit value of the reliability index, calculated from the characteristic width of the crack opening $A_{\text {lim; }} ; V_{\mathrm{R}}$ and $V_{\mathrm{Q}}$ - are generalized variation coefficient of the element and load, respectively.

\section{Conclusions}

A model based on the fundamental principles of a stochastic reliability theory is universal and suitable for all types of building constructions elements. The model in this case is guided by one parameter - the hazard rate. This fact is a decisive characteristic and makes the model a universal one. On the other hand, only one probability parameter is a disadvantage, narrows the capabilities of the model as a durability control device, and requires the researcher not standard, specific methods of a priori determination of the hazard rate. One of the effective ways to determine the hazard rate was proposed by us for the normative document on the service maintenance of highway bridges Ukrainian State Standard DSTU-N B V.2.3-23:2012 in A. Lantukh-Lyashchenko research works and determined by an expert on the basis of inspection of structures the facility in service. Clearly, in this case, a model in which reliability is a criterion for degradation can't be applied at the design stage.

The new model of prediction of the technical state of the elements of highway bridges is based on the analysis of the cracking resistance of bending reinforced concrete elements can be the most grounded, in terms of the physics destruction of reinforced concrete. It can be the basis for the development of a simple engineering apparatus for predicting the resource at all stages of the life cycle, from design. Obviously, due to the large number of physico-mechanical and geometric parameters included in the crack-width detection formula, the prediction of width opening cracking is the most well-founded form of analysis of durability.

The model opens the way of managing the resource by varying the mechanical characteristics of the materials. In the system of service maintenance of highway bridges, a model with a single control parameter, the width of crack opening, allows predicting the lifetime of reinforced concrete bridges elements at all stages of the life cycle of service, can greatly simplify the process of assessing their technical state. The disadvantage of this model is one - the model is suitable only for reinforced concrete elements.

\section{References}

1. M.A. Miner, Journal of Applied Mechanics 12(3), 159-164 (1945)

2. J.P. Dias, S. Ekwaro-Osire, A. Cunha, S. Dabetwar, A. Nispel, F.M. Alemayehu, H.B. Endeshaw, Parametric probabilistic approach for cumulative fatigue damage using double linear damage rule considering limited data 127, 246-258 (2019) https://doi.org/10.1016/j.ijfatigue.2019.06.011

3. Q. Li, C. Wang, B.R. Ellingwood, Struct. Saf. 52, 132-141 (2015) https://doi.org/10.1016/j.strusafe.2014.10.003

4. W.X. Yao, Acta Metallurgica Sinica 20(1), 65-71 (2007) ISSN 1006-7191, https://doi.org/10.1016/S1006-7191(07)60009-X.

5. F. Duprat, Construction and Building Materials 21(8), 1605-1616 (2007) ISSN 09500618, https://doi.org/10.1016/j.conbuildmat.2006.08.002

6. M. Torres-Luque, E. Bastidas-Arteaga, F. Schoefs, M. Sánchez-Silva, J.F. Osma, Constr. Build. $\quad$ Mater. $\quad$ 68, 68-81 https://doi.org/10.1016/j.conbuildmat.2014.06.009 
7. R.A. Atadero, G. Jia, A. Abdallah, M.E. Ozbek, Infrastructures 4, 50 (2019) https://doi.org/10.3390/infrastructures4030050

8. M.P. Enright, D.M. Frangopol, Eng. Struct. 20, 960-971 (1998) https://doi.org/10.1016/S0141-0296(97)00190-9

9. J.L. Bogdanoff, F. Kozin, Probabilistic models of cumulative damage (Wiley-Interscience, New York, 1985)

10. R.E. Melchers, Structural Reliability Analysis and Prediction (John Wiley \& Sons, Chichester, 1999)

11. ISO 2394. General principles on reliability for structures (International Organization for Standardization, Genève, 2015)

12. European Standard EN 1990:2002 Eurocode 1 (2001)

13. JCSS Probabilistic model code (singles file). Joint committee on structural safety (2001) ISBN 978-3-909386-79-6, https://www.jcss-lc.org/jcss-probabilistic-modelcode/

14. K. Aboura, B. Samali, K. Crews, J. Li, Stochastic Processes for Modeling Bridge Deterioration 6 (2009) https://www.researchgate.net/publication/281371822 _Stochastic_Processes_for_Modeling_Bridge_Deterioration

15. P. Bocchini, D. Saydam, D. Frangopol, Structural Safety 40, 51-64 (2013) https://doi.org/10.1016/j.strusafe.2012.09.004

16. M.J. Kallen, J.M. van Noortwijk, Third International Conference on Bridge Maintenance, Safety and Management (IABMAS), 535-536 (2006) https://doi.org/10.1201/b18175-219

17. S. Ranjith, S. Setunge, R. Gravina, S. Venkatesan, J. Perform. Constr. Facil. 27, 319-325 (2011) https://ascelibrary.org/doi/10.1061/\%28ASCE\%29CF.19435509.0000311

18. Z. Zhang, X. Sun, X. Wang, 9th International Bridge Management Conference (section Deterioration and Reliability), 207-219 (2003) http://onlinepubs.trb.org/onlinepubs/circulars/ec049.pdf 